\title{
STEADY STATES OF THE REACTION-DIFFUSION EQUATIONS. PART II: UNIQUENESS OF SOLUTIONS AND SOME SPECIAL CASES
}

\author{
J. G. BURNELL $L^{1}$, A. A. LACEY ${ }^{1,2}$ and G. C. WAKE'
}

(Received 9 July 1982)

\begin{abstract}
In an earlier paper (Part I) the existence and some related properties of the solution to a coupled pair of nonlinear elliptic partial differential equations was considered. These equations arise when material is undergoing an exothermic chemical reaction which is sustained by the diffusion of a reactant. In this paper we consider the range of parameters for which the uniqueness of solution is assured and we also investigate the converse question of multiple solutions. The special case of the one dimensional shape of the infinite slab is investigated in full as this case admits to solution by integration.
\end{abstract}

\section{Introduction}

In Part I of this paper (Burnell, Lacey and Wake [3]) we considered the steady-states of the equations governing the diffusion of a reactant which is undergoing an exothermic reaction. This led to the equations (4), (5) of that paper, that is:

$$
\begin{array}{cc}
\nabla^{2} u+\lambda(1+v) e^{u}=0 & \text { in } \Omega, \\
\nabla^{2} v-\alpha \lambda(1+v) e^{u}=0 & \text { in } \Omega,
\end{array}
$$

with

$$
\begin{array}{ll}
\partial u / \partial n+\mu u=0 & \text { on } \partial \Omega, \\
\partial v / \partial n+\nu v=0 & \text { on } \partial \Omega,
\end{array}
$$

\footnotetext{
'Mathematics Department, Victoria University, Private Bag, Wellington, New Zealand. Please address all correspondence to Dr. Wake at this address.

${ }^{2}$ Present address: Mathematics Department, Heriot-Watt University, Edinburgh EH14 4AS, Scotland. (1) Copyright Australian Mathematical Society 1983
} 
where $\Omega \subseteq \mathbf{R}^{n}$ and $\lambda, \alpha, \mu, \nu$ are constants $(\lambda, \alpha>0,0<\mu, \nu \leqslant \infty)$. If $\mu$ or $\nu$ are infinite then the condition in (2) becomes the appropriate Dirichlet boundary condition.

Part I establishes the existence of solutions to these equations for all values of the parameters except when $\mu<\nu=\infty$. In fact we showed that there exist solutions for the more general system of equations

$$
\begin{gathered}
\nabla^{2} u+\lambda f(u, v)=0 \quad \text { in } \Omega, \\
\nabla^{2} v-\alpha \lambda f(u, v)=0 \quad \text { in } \Omega,
\end{gathered}
$$

with boundary conditions (2) on $\partial \Omega$, where $f(u, v)$ has the properties:

a) there is some $v_{0}<0$ such that $f\left(u, v_{0}\right)=0$ for all $u \in \mathbf{R}$;

b) $f(u, \cdot): y \mapsto f(u, y)$ and $f(\cdot, v): x \mapsto f(x, v)$ are monotonic increasing for all $u \in \mathbf{R}$ and $v \geqslant v_{0}$ respectively;

c) $f \in C^{1}\left(\mathbf{R}^{2}\right)$.

Further we established bounds on the solutions,

$$
\begin{aligned}
v_{0} & \leqslant v(x) \leqslant 0, \\
0 & \leqslant u(x) \leqslant-v_{0} / \alpha \quad \text { for } \nu \leqslant \mu, \\
0 & \leqslant u(x) \leqslant-v_{0} \nu / \alpha \mu \text { for } \mu<\nu<\infty .
\end{aligned}
$$

For the case of $\mu<\nu=\infty$ and $n \leqslant 3$ we showed that there exists a value of $\lambda$, say $\lambda^{*}$, such that (1), (2) has a solution for $\lambda<\lambda^{*}$, and no solution for $\lambda>\lambda^{*}$. The last section of Part I established that where solutions are unique the solution branch $(\lambda,(u, v))$ is continuous in $\lambda$.

In this paper we shall find some sufficient conditions so that the problem (1), (2) (or occasionally (2), (3)) has a unique solution for some values of $\lambda$. For a special class of problems we also show that multiple solutions exist for a particular range of the parameters $\lambda, \alpha, \mu$, and $\nu$. We conclude this Part by discussing the case when $n=1$, that is $\Omega=(-1,1)$, which is a representation of an infinite slab with parallel sides.

\section{Uniqueness of solutions}

We begin by noting that for the special case $\mu=\nu$ for which the harmonic function $h \equiv \alpha u+v$ is zero and (2), (3) reduces to the simpler system

$$
\begin{aligned}
\nabla^{2} u+\lambda f(u,-\alpha u)=0 & \text { in } \Omega, \\
\partial u / \partial n+\mu u=0 & \text { on } \partial \Omega
\end{aligned}
$$


there is the following result (Aris [2]):

LEMMA 1. If $f(u,-\alpha u) / u$ is a positive decreasing function of $u$ for $u>0$, there is at most one solution to (2), (3) for all values of $\lambda \geqslant 0$.

For the problem with $f(u, v)=(1+v) e^{u}$ this tells us that this special case of

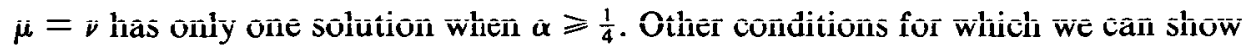
uniqueness are for small $\lambda$ with $\nu<\infty$ or $\mu=\nu=\infty$, or large $\lambda$ with $\nu<\infty$. We shall make use of contraction mappings in each case.

From the inequalities (4) we can easily show uniqueness for a range of $\lambda$.

THEOREM 2. For $\mu=\nu=\infty$ or $\nu<\infty$ the system (2), (3) has a unique solution for sufficiently small $\lambda$.

Proof. We again consider the map $T: C(\bar{\Omega}) \times C(\bar{\Omega}) \rightarrow C(\bar{\Omega}) \times C(\bar{\Omega}), \quad T$ : $(u, v) \mapsto\left(\lambda K_{\mu} F(u, v),-\alpha \lambda K_{v} F(u, v)\right)$ defined in Part I, Section 2 (Burnell, Lacey and Wake [3]), where $K_{\mu}, K_{\nu}$ are the extensions to $C(\bar{\Omega})$ of the solution operators for $\left(-\nabla^{2}, \partial / \partial n+\mu\right),\left(-\nabla^{2}, \partial / \partial n+\nu\right)$ respectively. That is, for $g \in$ $C(\bar{\Omega}), K_{\mu} g\left(K_{\nu} g\right)$ is the unique solution of

$$
\begin{gathered}
-\nabla^{2} w=g \quad \text { in } \Omega, \\
\partial w / \partial n+\mu w=0 \quad(\partial w / \partial n+\nu w=0) \quad \text { on } \partial \Omega .
\end{gathered}
$$

As in Part I we consider a ball of radius $r=-v_{0}(1+\max \{1, \nu / \mu\} / \alpha)$, that is

$$
B=\left\{(u, v) \in C(\bar{\Omega}) \times C(\bar{\Omega}):\|u\|_{0}+\|v\|_{0}<r\right\}
$$

where, if $\mu=\nu=\infty$, we take 1 in place of $\nu / \mu$.

Now any fixed point of $T$ is a solution of (2), (3) and vice versa, so we wish to show uniqueness of the fixed points of $T$ (see Part I, Section 2, Proposition 5). For $\lambda$ sufficiently small, it follows that $T$ is a contraction mapping on $\bar{B}$ as established in Part I, Section 3. We know by the inequalities in (4) that any solution of (2), (3) must be in $\bar{B}$. Hence by the contraction mapping theorem there is only one fixed point of $T$ in $\bar{B}$ for sufficiently small $\lambda$. Hence the solution is unique for small $\lambda$. Q.E.D.

This proof again depends on the boundedness of $\nu / \mu$. For the case of $\mu<\nu=\infty$ we shall see later in Section 3 that (2), (3) has multiple solutions for arbitrarily small values of $\lambda$ in the one-dimensional case $(n=1)$. We expect similar behaviour for other regions $\Omega$.

Next we shall examine the uniqueness of solutions for large $\lambda$. The only results we have obtained are for $\nu<\infty$, although asymptotic analysis of the case when 
$\mu=\nu=\infty$ strongly suggests a similar result in this case. Of course for $\mu<\nu=\infty$, (2), (3) fails to have any solutions for sufficiently large $\lambda$.

We shall only prove the result for the particular case $f(u, v)=(1+v) e^{u}$, although the proof can be generalised to cover arbitrary $f$ satisfying our previously specified conditions.

The above inequalities (4) for $v$ and $u$ are applied again, together with those for the harmonic function $h=\alpha u+v$ :

$$
\begin{aligned}
-1+\nu / \mu \leqslant h(x) \leqslant 0 & \text { for } \nu \leqslant \mu, \\
0 \leqslant h(x) \leqslant \nu / \mu-1 & \text { for } \mu \leqslant \nu<\infty .
\end{aligned}
$$

We proceed by first refining the inequalities in (4), (6) for all cases of $\nu<\infty$.

Lemma 3. Suppose $(u, v)$ satisfies (1), (2); then there exists a positive constant $A$ such that for $\lambda$ sufficiently large

$$
-1 \leqslant v(x) \leqslant-1+A(\alpha \lambda)^{-1 / 2}, \quad \forall x \in \bar{\Omega} .
$$

Proof. The result will be proved by constructing a suitable upper solution for $v$. Let $\phi$ satisfy

$$
\nabla^{2} \phi+1=0 \text { in } \Omega ; \quad \phi=0 \text { on } \partial \Omega .
$$

By the result in Part I, Section 2, Proposition $1, \phi \in C^{2+\sigma}(\bar{\Omega}), 0 \leqslant \sigma<1$, so we define

$$
k=1+\max _{x \in \bar{\Omega}}|\nabla \phi| .
$$

We then let $w=\phi / k$ and $v_{1}=A(\alpha \lambda)^{-1 / 2} \exp \left[-(\alpha \lambda)^{1 / 2} w\right]-1$ where $A=$ $\nu / \min _{x \in \partial \Omega}\{-\partial w / \partial n\}$. It follows from the maximum principle that $\partial w / \partial n$ is negative and bounded away from zero so that $A$ is well-defined and positive.

We now show that $v_{1}$ is an upper solution for (lb). Firstly $\nabla^{2} v_{1}=$ $(\alpha \lambda)^{1 / 2} A \exp \left(-(\alpha \lambda)^{1 / 2} w\right)|\nabla w|^{2}-A \exp \left(-(\alpha \lambda)^{1 / 2} w\right) \nabla^{2} w$. So for $\lambda>k^{-2} \alpha^{-1}(1$ $\left.-\max _{x \in \bar{\Omega}}|\nabla w|^{2}\right)^{-2}$ (which is well-defined by the definitions of $k$ and $w$ ) we have, since $u \geqslant 0$,

$$
\begin{aligned}
\nabla^{2} v_{1}-\alpha \lambda(1 & \left.+v_{1}\right) e^{u} \\
& =A \exp \left(-(\alpha \lambda)^{1 / 2} w\right)\left[(\alpha \lambda)^{1 / 2}|\nabla w|^{2}-\nabla^{2} w-(\alpha \lambda)^{1 / 2} e^{u}\right] \\
& \leqslant A \exp \left(-(\alpha \lambda)^{1 / 2} w\right)(\alpha \lambda)^{1 / 2}\left[|\nabla w|^{2}+(\alpha \lambda)^{-1 / 2} / k-1\right]<0 .
\end{aligned}
$$

Secondly, since $w=0$ and $\partial w / \partial n<0$ on $\partial \Omega$,

$$
\begin{aligned}
\frac{\partial v_{1}}{\partial n}+\nu v_{1} & =A \exp \left(-(\alpha \lambda)^{1 / 2} w\right)\left(-\frac{\partial w}{\partial n}+\nu(\alpha \lambda)^{-1 / 2}\right)-\nu \\
& =\nu\left[-\frac{\partial w}{\partial n} / \min _{x \in \partial \Omega}\left\{-\frac{\partial w}{\partial n}\right\}-1+A(\alpha \lambda)^{-1 / 2}\right] \geqslant 0 .
\end{aligned}
$$


Consequently $v_{1}$ is an upper solution for (lb); while -1 is a lower solution. Therefore there is some solution lying between -1 and $v_{1}$ (Sattinger [5, Theorem 2.3.1]). But we have already shown that the solution to (lb) for a given $u$ is unique (see Part I, Section 3, Lemma 1), so $-1 \leqslant v(x) \leqslant v,(x), x \in \bar{\Omega}$. Since $\min \{w(x)$ : $x \in \bar{\Omega}\}=0$, it follows that

$$
-1 \leqslant v(x) \leqslant-1+(\alpha \lambda)^{-1 / 2} A \text { for } x \in \bar{\Omega} .
$$

We can easily prove the following corollary, using the equations satisfied by $h=\alpha u+v$ :

$$
\begin{aligned}
& \nabla^{2} h=0 \quad \text { in } \Omega, \\
& \frac{\partial h}{\partial n}+\mu h=(\mu-\nu) v \quad \text { on } \partial \Omega .
\end{aligned}
$$

Corollary -4. Suppose $(u, v)$ satisfies (1), (2) and $h=\alpha u+v$. Then for sufficiently large $\lambda$,

$$
\begin{gathered}
-1+\nu / \mu \leqslant h(x) \leqslant-1+\nu / \mu+(1-\nu / \mu)(\alpha \lambda)^{-1 / 2} A, \\
\left(\nu / \mu-(\alpha \lambda)^{-1 / 2} A\right) / \alpha \leqslant u(x) \leqslant \nu / \mu+(\alpha \lambda)^{-1 / 2}(1-\nu / \mu) A, \\
\forall x \in \bar{\Omega} \quad \text { if } \mu \geqslant \nu ; \\
-1+\nu / \mu-(\alpha \lambda)^{-1 / 2}(\nu / \mu-1) \leqslant h(x) \leqslant \nu / \mu-1, \\
\nu\left(1-(\alpha \lambda)^{-1 / 2} A\right) / \alpha \mu \leqslant u(x) \leqslant \nu / \alpha \mu, \quad \forall x \in \bar{\Omega} \quad \text { if } \mu \leqslant \nu<\infty .
\end{gathered}
$$

These inequalities allow us to prove the result given in Theorem 5.

THEOREM 5. If $\nu<\infty$ then for $\lambda$ sufficiently large there is at most one solution to (1), (2).

Proof. We shall construct a map $S$ the fixed points of which are precisely the solutions of (1), (2). Next we shall show that $S$ has at most one fixed point for sufficiently large $\lambda$. We present the proof for $\nu<\mu \leqslant \infty$ only, as for the other case, $\mu \leqslant \nu<\infty$, the proof is similar and is left to the reader.

Use will be made of the equations satisfied by $h=\alpha u+v$ and $v$, that is (8) and

$$
\begin{aligned}
\nabla^{2} v-\lambda \alpha(1+v) e^{(h-v) / \alpha}=0 & \text { in } \Omega \\
\frac{\partial v}{\partial n}+\nu v=0 & \text { on } \partial \Omega
\end{aligned}
$$


For $h, v \in C^{2}(\Omega) \cap C^{1}(\bar{\Omega})$ we define $S(h, v)$ to be the pair $\left(h^{\prime}, v^{\prime}\right)$ where $v^{\prime}$ is the unique solution of

$$
\begin{gathered}
\nabla^{2} v^{\prime}-\alpha \lambda e^{\nu / \alpha \mu} v^{\prime}=\alpha \lambda(1+v) e^{(h-v) / \alpha}-\alpha \lambda e^{\nu / \alpha \mu} v \text { on } \Omega, \\
\frac{\partial v^{\prime}}{\partial n}+\nu v^{\prime}=0 \text { on } \partial \Omega,
\end{gathered}
$$

and $h^{\prime}$ is the (unique) solution of

$$
\nabla^{2} h^{\prime}=0 \quad \text { in } \Omega, \quad \partial h^{\prime} / \partial n+\mu h^{\prime}=(\mu-\nu) v^{\prime} \quad \text { on } \partial \Omega .
$$

(Again for $\nu<\mu=\infty$ the boundary condition reduces to $h^{\prime}=v^{\prime}$ on $\partial \Omega$.)

Now suppose that $h_{j}, v, \in C^{2}(\Omega) \cap C^{1}(\bar{\Omega}), h_{j}$, satisfies (9a), and $v$, satisfies (7) for $j=1,2$. Then we have

$$
\begin{aligned}
& \left(\nu / \mu-(\alpha \lambda)^{-1 / 2} A\right) / \alpha \leqslant\left(h_{j}(x)-v_{j}(x)\right) / \alpha \\
& \quad \leqslant\left(\nu / \mu-A(1-\nu / \mu)(\alpha \lambda)^{-1 / 2}\right) / \alpha \text { for } x \in \bar{\Omega} \text { and } j=1,2 .
\end{aligned}
$$

Letting $\left(h_{j}^{\prime}, v_{j}^{\prime}\right)=S\left(h_{j}, v_{j}\right)$ we obtain

$$
\begin{aligned}
& \nabla^{2}\left(v_{1}^{\prime}-v_{2}^{\prime}\right)-\alpha \lambda e^{\nu / \alpha \mu}\left(v_{1}^{\prime}-v_{2}^{\prime}\right) \\
& \quad=\alpha \lambda\left\{\left(1+v_{1}\right) e^{\left(h_{1}-v_{1}\right) / \alpha}-\left(1+v_{2}\right) e^{\left(h_{2}-v_{2}\right) / \alpha}+e^{\nu / \alpha \mu}\left(v_{1}-v_{2}\right)\right\},
\end{aligned}
$$

or

$$
\begin{aligned}
& \nabla^{2}\left(v_{1}^{\prime}-v_{2}^{\prime}\right)-\alpha \lambda e^{\nu / \alpha \mu}\left(v_{1}^{\prime}-v_{2}^{\prime}\right) \\
& \quad=\alpha \lambda\left\{\left(v_{1}-v_{2}\right)\left(e^{\left(h_{1}-v_{1}\right) / \alpha}-e^{\nu / \alpha \mu}\right)+\left(1+v_{2}\right)\left(e^{\left(h_{1}-v_{1}\right) / \alpha}-e^{\left(h_{2}-v_{2}\right) / \alpha}\right)\right\} .
\end{aligned}
$$

Now $e^{\left(h_{1}-v_{1}\right) / \alpha}-e^{\left(h_{2}-v_{2}\right) / \alpha}=e^{\zeta}\left(\left(h_{1}-h_{2}\right)+\left(v_{2}-v_{2}\right)\right) / \alpha$ where $\zeta(x)$ lies between $\left(h_{1}(x)-v_{1}(x)\right) / \alpha$ and $\left(h_{2}(x)-v_{2}(x)\right) / \alpha$ for all $x \in \bar{\Omega}$. Since $\mu \geqslant \nu$ it follows from (12) that for $\lambda$ sufficiently large,

$$
0 \leqslant\left(h_{j}(x)-v_{j}(x)\right) / \alpha \leqslant 1 / \alpha \text { for } x \in \bar{\Omega} \text { and } j=1,2,
$$

and so

$$
\zeta(x) \leqslant 1 / \alpha \text { for } x \in \bar{\Omega} .
$$

Consequently

$$
\begin{aligned}
-\left(\left|h_{1}-h_{2}\right|+\left|v_{1}-v_{2}\right|\right) e^{1 / \alpha} / \alpha & \leqslant e^{\left(h_{1}-v_{1}\right) / \alpha}-e^{\left(h_{2}-v_{2}\right) / \alpha} \\
& \leqslant\left(\left|h_{1}-h_{2}\right|+\left|v_{1}-v_{2}\right|\right) e^{1 / \alpha} / \alpha .
\end{aligned}
$$

Also,

$$
e^{\left(h_{1}-v_{1}\right) / \alpha}-e^{\nu / \alpha \mu}=e^{\xi}\left(h_{1}-v_{1}-\nu / \mu\right) / \alpha,
$$


where $\xi(x)$ lies between $\left(h_{1}(x)-v_{1}(x)\right) / \alpha$ and $\nu / \alpha \mu$ for all $x \in \bar{\Omega}$. But, using (12), we obtain

$$
\begin{aligned}
-(\alpha \lambda)^{-1 / 2} A e^{1 / \alpha} / \alpha & \leqslant e^{\left(h_{1}-v_{1}\right) / \alpha}-e^{\nu / \alpha \mu} \\
& \leqslant(\alpha \lambda)^{-1 / 2} A e^{1 / \alpha} / \alpha
\end{aligned}
$$

Finally

$$
0 \leqslant 1+v_{2}(x) \leqslant(\alpha \lambda)^{-1 / 2} A \text { for } x \in \bar{\Omega} .
$$

Putting (14), (15), (16) into (13) we obtain

$$
\begin{aligned}
-(\alpha \lambda)^{1 / 2}\left(A e^{1 / \alpha} / \alpha\right)\left(2\left|v_{1}-v_{2}\right|+\left|h_{1}-h_{2}\right|\right) & \\
& \leqslant-\nabla^{2}\left(v_{2}^{\prime}-v_{1}^{\prime}\right)+\alpha \lambda e^{\nu / \alpha \mu}\left(v_{2}^{\prime}-v_{1}^{\prime}\right) \\
& \leqslant(\alpha \lambda)^{1 / 2}\left(A e^{1 / \alpha} / \alpha\right)\left(2\left|v_{1}-v_{2}\right|+\left|h_{1}-h_{2}\right|\right) \quad \text { in } \Omega .
\end{aligned}
$$

Applying the maximum principle for the operator $-\nabla^{2}+\alpha \lambda e^{\nu / \alpha \mu}$, we obtain

$$
\left|v_{1}^{\prime}-v_{2}^{\prime}\right| \leqslant(\alpha \lambda)^{-1 / 2}\left(A e^{(1-\nu / \mu) / \alpha} / \alpha\right)\left(2\left\|v_{1}-v_{2}\right\|_{0}+\left\|h_{1}-h_{2}\right\|_{0}\right) \text { in } \bar{\Omega} \text {. }
$$

Also $\nabla^{2}\left(h_{2}^{\prime}-h_{1}^{\prime}\right)=0$ in $\Omega$,

$$
\frac{\partial}{\partial n}\left(h_{2}^{\prime}-h_{1}^{\prime}\right)+\mu\left(h_{2}^{\prime}-h_{1}^{\prime}\right)=(\mu-\nu)\left(v_{2}^{\prime}-v_{1}^{\prime}\right) \quad \text { on } \partial \Omega \text {. }
$$

Hence $\left|h_{1}^{\prime}-h_{2}^{\prime}\right| \leqslant(1-\nu / \mu)\left\|v_{1}^{\prime}-v_{2}^{\prime}\right\|_{0}$.

Therefore

$$
\begin{aligned}
\left\|v_{2}^{\prime}-v_{1}^{\prime}\right\|_{0}+\left\|h_{2}^{\prime}-h_{1}^{\prime}\right\|_{0} \leqslant & (\alpha \lambda)^{-1 / 2}\left\{(2-\nu / \mu) A e^{(1-\nu / \mu) / \alpha} / \alpha\right\} \\
& \times\left\{2\left\|v_{1}-v_{2}\right\|_{0}+\left\|h_{1}-h_{2}\right\|_{0}\right\} \\
\leqslant & (\alpha \lambda)^{-1 / 2}\left\{2(2-\nu / \mu) A e^{(1-\nu / \mu) / \alpha} / \alpha\right\} \\
& \times\left(\left\|v_{2}-v_{1}\right\|_{0}+\left\|h_{2}-h_{1}\right\|_{0}\right) .
\end{aligned}
$$

We now take $\lambda$ large enough so that any solution $(u, v)$ to (1), (2) satisfies Lemma 3 and

$$
\lambda \geqslant\left(4(2-\nu / \mu) A e^{(1-\nu / \mu) / \alpha}\right)^{2} / \alpha^{3}
$$

Suppose that $\left(u, v_{j}\right)$ for $j=1,2$ are solutions of $(1),(2)$ for such a value of $\lambda$. Then $\left(h_{\jmath}, v_{\jmath}\right)$, where $h_{\jmath}=\alpha u_{\jmath}+v_{\jmath}$, are solutions of (8), (11) and so are fixed points of $S$. Also $h, v$, satisfy (7), (9a), so that (17) holds. Hence

$$
\left\|v_{2}-v_{1}\right\|_{0}+\left\|h_{2}-h_{1}\right\|_{0} \leqslant \frac{1}{2}\left(\left\|v_{2}-v_{1}\right\|_{0}+\left\|h_{2}-h_{1}\right\|_{0}\right) \text {, }
$$

since $v_{j}^{\prime}=v$, and $h_{j}^{\prime}=h_{j}$. For this inequality to hold $\left(v_{2}, h_{2}\right)=\left(v_{1}, h_{1}\right)$, that is, there is at most one solution. Q.E.D. 
In Part I we showed that for $\nu<\infty$ or $\mu=\nu=\infty$, (1), (2) has a solution for all values of $\lambda$. So using Theorem 5, we see that for $\nu<\infty$ the solution is unique for $\lambda$ sufficiently small or sufficiently large. For the case $\mu=\nu=\infty$, by Theorem 2 , there is a unique solution for $\lambda$ sufficiently small. In this instance $(\mu=\nu=\infty)$ our method fails to prove uniqueness for large $\lambda$ since $v$ always vanishes on $\partial \Omega$ and we cannot obtain the key estimates

$$
v \leqslant-1+(\alpha \lambda)^{-1 / 2} A, \quad\left(1-(\alpha \lambda)^{-1 / 2} A\right) / \alpha \leqslant u \leqslant 1 / \alpha .
$$

In Part I, Section 3 we showed that if there is a unique solution for $\lambda$ in an open interval, then the solution is continuous in $\lambda$. The previous results (such as that in Theorem 5) show that such cases arise. In particular, if $\nu<\infty$ there exists a continuous branch of solutions on some interval $\left(\lambda_{1}, \infty\right)$.

We conclude this section by demonstrating the occurrence of multiple solutions for $\alpha$ sufficiently small when $\mu=\nu$, in which case the equations (1), (2) are uncoupled to give

$$
\left.\begin{array}{ll}
\nabla^{2} u+\lambda(1-\alpha u) e^{u}=0 & \text { in } \Omega, \\
\frac{\partial u}{\partial n}+\mu u=0 & \text { on } \partial \Omega .
\end{array}\right\}
$$

For $\alpha=0$ the system further simplifies to

$$
\begin{array}{ll}
\nabla^{2} u_{0}+\lambda e^{u_{0}}=0 & \text { in } \Omega, \\
\frac{\partial u_{0}}{\partial n}+\mu u_{0}=0 & \text { on } \partial \Omega .
\end{array}
$$

It is known that this last problem has solutions for $\lambda$ small, say $\lambda<\lambda_{0}$ (Keller and Cohen [4], Amann [1]). If we take such a value of $\lambda$ we find that

$$
\nabla^{2} u_{0}+\lambda\left(1-\alpha u_{0}\right) e^{u_{0}} \leqslant 0 \text { in } \Omega,
$$

together with the same boundary conditions, which means that $u_{0}$ is an upper solution for (18). Once again $u \equiv 0$ is a lower solution. Hence there is a solution $u_{1}$ of (18) with $0<u_{1}<u_{0}$. (We note that the strong maximum principle ensures that $u \equiv 0$ and $u=u_{0}$ are, in fact strict lower and upper solutions respectively.)

Taking a function $g \in C^{2}(\bar{\Omega})$ such that $g$ is nonnegative with nonempty support $D \subset \Omega$, we define $u_{s}$ by

$$
\begin{array}{ll}
\nabla^{2} u_{s}+g=0 & \text { in } \Omega, \\
\frac{\partial u_{s}}{\partial n}+\mu u_{s}=0 & \text { on } \partial \Omega .
\end{array}
$$

We shall consider the function $C u_{s}$, where $C$ is a positive constant still to be chosen. Now

$$
\dot{\nabla}^{2}\left(C u_{s}\right)+\lambda\left(1-\alpha C u_{s}\right) e^{C u_{s}}=\lambda\left(1-\alpha C u_{s}\right) e^{C u_{s}}-C g
$$


We take $C$ to be sufficiently large so that:

(a) $\forall x \in \Omega, \lambda e^{C u_{s}}-C g \geqslant B>0$.

(Clearly this is possible since $\inf _{x \in D}\left\{u_{s}(x)\right\}>0$ and $g=0$ for $x \notin D$.)

(b) $C\left\|u_{s}\right\|_{0}>\left\|u_{0}\right\|_{0}$.

We now choose $\alpha$ sufficiently small so that

$$
\alpha \lambda C\left\|u_{s}\right\|_{0} \exp \left(C\left\|u_{s}\right\|_{0}\right) \leqslant B .
$$

Dy (19) $C u_{s}$ is now a lower solutition for (iô) (ine right hand side of (ị) is positive). Moreover if, in addition,

$$
\alpha \leqslant\left(C\left\|u_{s}\right\|_{0}\right)^{-1},
$$

then $u \equiv 1 / \alpha$, which is clearly an upper solution for (18), is greater than $C u_{s}$. We deduce that there is a solution $u_{2}$ of (18) with $C u_{s} \leqslant u_{2} \leqslant 1 / \alpha$. As $\left\|u_{0}\right\|_{0}<$ $C\left\|u_{s}\right\|_{0}$ and $C u_{s} \leqslant u_{2}$ on $\bar{\Omega}$ this means $u_{1} \neq u_{2}$ and we have established that there are at least two distinct solutions to (18) for these values of $\lambda$ and $\alpha$.

\section{The special case of the infinite slab}

One particular example for which we can obtain extra information about the solution set of the problem (1), (2) is the case when $\Omega$ is a subset of $\mathbf{R}$. Such a region $\Omega$ may be thought of as representing an infinite parallel sided slab. Without loss of generality we can take the sides of the slab to be at $x= \pm 1$. Once again we shall only consider the function $f$ given by $f(u, v)=(1+v) e^{u}$, although many of the following results apply to more general functions $f$.

The problem (1), (2) now becomes

$$
\begin{aligned}
d^{2} u / d x^{2}+\lambda(1+v) e^{u}=0 & \text { for }-1<x<1, \\
d^{2} v / d x^{2}-\lambda \alpha(1+v) e^{u}=0 & \text { for }-1<x<1,
\end{aligned}
$$

and

$$
\begin{aligned}
& \frac{d u}{d x}( \pm 1) \pm \mu u( \pm 1)=0, \\
& \frac{d v}{d x}( \pm 1) \pm v v( \pm 1)=0 .
\end{aligned}
$$

In order to proceed with the calculations of the problem (20), (21) we will only consider solutions $(u, v)$ which are symmetric on $\Omega=(-1,1)$. Then the boundary conditions (21) become

$$
\frac{d u}{d x}(1)+\mu u(1)=0, \quad \frac{d u}{d x}(0)=0,
$$


and

$$
\frac{d v}{d x}(1)+v v(1)=0, \quad \frac{d v}{d x}(0)=0 .
$$

In fact, if $\nu \leqslant \mu$ then any solution to (20), (21) will be symmetric (for details see Aris [2]). Further, when $\mu<\nu$ we showed in Part I (see Note in Section 3) that there is a minimal solution $\left(u_{m}, h_{m}\right)$ to the problem

$$
\begin{aligned}
& \left.\begin{array}{l}
d^{2} u / d x^{2}+\lambda(1+h-\alpha u) e^{u}=0 \text { for }-1<x<1, \\
\frac{d u}{d x}( \pm 1) \pm \mu u( \pm 1)=0,
\end{array}\right\} \\
& \left.\begin{array}{l}
d^{2} h / d x^{2}=0 \text { for }-1<x<1 \\
\frac{d h}{d x}( \pm 1) \pm \nu h( \pm 1)=\alpha(\mu-\nu) u( \pm 1)
\end{array}\right\}
\end{aligned}
$$

If $u_{1}(x)=u_{m}(-x)$ and $h_{1}(x)=h_{m}(-x)$ then $\left(u_{1}, h_{1}\right)$ is also a solution to (23) and so $(\forall x \in(-1,1)) u_{1}(x) \geqslant u_{m}(x)$. Hence $(\forall x \in(-1,1)) u_{m}(-x) \geqslant u_{m}(x)$, so $u_{m}$ must be symmetric. The symmetry of $h_{m}$, and hence of $v_{m}=h_{m}-\alpha u_{m}$, follows similarly. Likewise one can show that the maximal solution to (23) is symmetric.

The system (20), (22) can be considered as representing an infinite parallel sided slab with one surface $(x=0)$ insulated and impermeable to the reactant, so that there is zero heat and mass flux across $x=0$.

The first special case which we shall solve is the double-Dirichlet problem, $\mu=\nu=\infty$. Here $v=-\alpha u$ and the system reduces to

$$
\begin{gathered}
d^{2} u / d x^{2}+\lambda(1-\alpha u) e^{u}=0 \text { for } 0<x<1, \\
\frac{d u}{d x}(0)=u(1)=0 .
\end{gathered}
$$

This can be integrated once to yield

$$
(d u / d x)^{2}=2 \lambda\left[G(u(x))-G\left(u_{0}\right)\right],
$$

where $G(u)=(1+\alpha-\alpha u) e^{u}$ and $u_{0}=u(0)$ which will be the maximum value of $u$.

Using the boundary condition $u(1)=0$, we obtain on further integration

$$
\sqrt{2 \lambda}=\int_{0}^{u_{0}}\left[G\left(u_{0}\right)-G(s)\right]^{-1 / 2} d s .
$$

We now define the function $I(y)$ by

$$
I(y)=\int_{0}^{y}[G(y)-G(s)]^{-1 / 2} d s,
$$

and proceed to examine its properties. These will be found to depend crucially on the value of $\alpha$, which appears as a parameter in the function $G$. For the rest of 
this section we will denote differentiation by ' - that is

$$
g^{\prime}(x)=\frac{d}{d x} g(x)
$$

where $g$ is a function $\mathbf{R} \rightarrow \mathbf{R}$.

Now, the graphs of $G, G^{\prime}, G^{\prime \prime}$ are as shown in Figures 1 and 2.

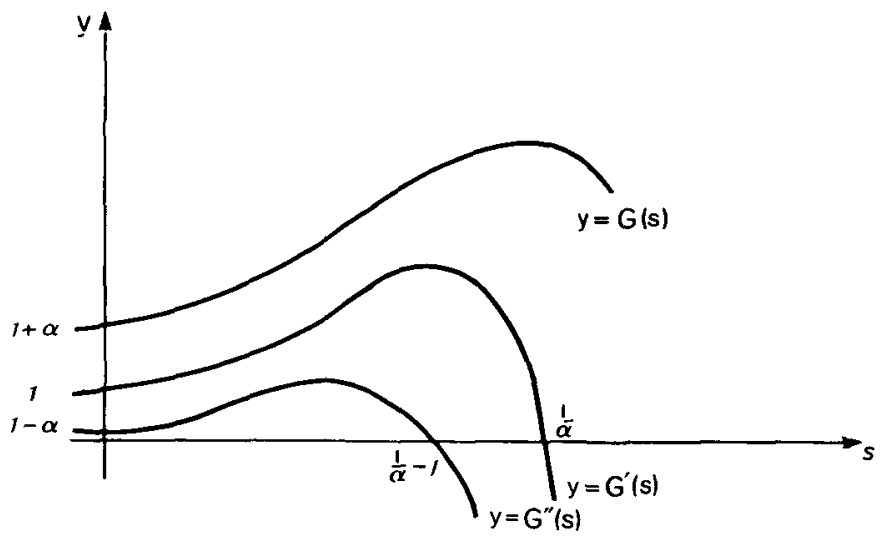

Figure 1. Graphs of $G, G^{\prime}$ and $G^{\prime \prime}$ for $\alpha<1$.

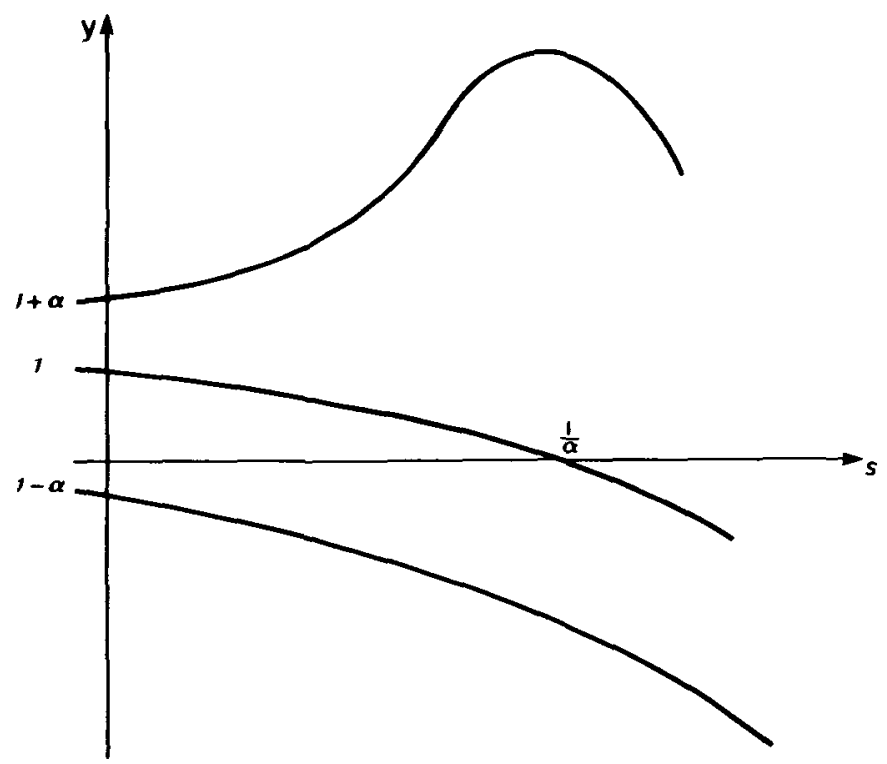

FIGURE 2. Graphs of $G, G^{\prime}$ and $G^{\prime \prime}$ for $\alpha \geq 1$. 
Consequently we have, for $0 \leqslant s \leqslant y$,

$$
G(y)-G(s)=G^{\prime}(\zeta(s))(y-s) \geqslant A(y)(y-s),
$$

where $s \leqslant \zeta(s) \leqslant y$ and $A(y)=\min \left\{1, G^{\prime}(y)\right\}$.

This inequality shows that $I(y)$ is well-defined for any $y \in[0,1 / \alpha)$. Clearly $I(y)$ is not defined for $y>1 / \alpha$. Let us also note that $I(0)=0$.

Firstly we consider the behaviour of $I(y)$ for $y$ near $1 / \alpha$.

LeMmA 1. For any $y_{1}, y_{2}$ such that $1 / \alpha-1<y_{1}<y_{2}<1 / \alpha$, if $y_{2}<y<1 / \alpha$ then

$$
\begin{aligned}
& (2 / k(y))^{1 / 2} \cosh ^{-1}[(\alpha+\alpha y-1) y /(1-\alpha y)+1] \leqslant I(y) \\
& \leqslant\left(2 / k\left(y_{1}\right)\right)^{1 / 2} \cosh ^{-1}\left[k\left(y_{1}\right)\left(y-y_{1}\right) / G^{\prime}(y)+1\right]+l\left(y_{1}, y_{2}\right),
\end{aligned}
$$

where

$$
k(y)=-G^{\prime \prime}(y)=(\alpha+\alpha y-1) e^{y},
$$

and

$$
l\left(y_{1}, y_{2}\right)=y_{1}\left[G\left(y_{2}\right)-G\left(y_{1}\right)\right]^{-1 / 2} \text {. }
$$

Further

$$
I(y) \sim\left(2 e^{-1 / \alpha} / \alpha\right)^{1 / 2} \ln [1 /(1-\alpha y)] \text { as } y \rightarrow 1 / \alpha .
$$

Proof. From Figures 1 and 2 we have, for $y>1 / \alpha-1$ and $0 \leqslant s<y$,

$$
\begin{aligned}
G(y)-G(s) & =G^{\prime}(y)(y-s)-\frac{1}{2} G^{\prime \prime}(\zeta(s))(y-s)^{2} \quad(\text { where } s \leqslant \zeta(s) \leqslant y) \\
& \leqslant G^{\prime}(y)(y-s)+\frac{1}{2} k(y)(y-s)^{2},
\end{aligned}
$$

since $G^{\prime \prime}(\zeta(s)) \geqslant G^{\prime \prime}(y)=-k(y)$. Therefore

$$
\begin{aligned}
I(y) & \geqslant \int_{0}^{y}\left[G^{\prime}(y)(y-s)+\frac{1}{2} k(y)(y-s)^{2}\right]^{-1 / 2} d s \\
& =(2 / k(y))^{1 / 2} \cosh ^{-1}\left[1+k(y) y / G^{\prime}(y)\right],
\end{aligned}
$$

which is the left hand inequality.

To prove the other inequality we partition the interval $[0, y]$ into the intervals $\left[0, y_{1}\right]$ and $\left[y_{1}, y\right]$. On the latter interval

$$
\begin{aligned}
G(y)-G(s) & =G^{\prime}(y)(y-s)-\frac{1}{2} G^{\prime \prime}(\zeta(s))(y-s)^{2} \\
& \geqslant G^{\prime}(y)(y-s)+\frac{1}{2} k\left(y_{1}\right)(y-s)^{2},
\end{aligned}
$$


where $s \leqslant \zeta(s) \leqslant y$. Hence

$$
\begin{aligned}
\int_{y_{1}}^{y}[G(y)-G(s)]^{-1 / 2} d s & \leqslant \int_{y_{1}}^{y}\left[G^{\prime}(y)(y-s)+\frac{1}{2} k\left(y_{1}\right)(y-s)^{2}\right]^{-1 / 2} d s \\
& =\left(2 / k\left(y_{1}\right)\right)^{1 / 2} \cosh ^{-1}\left[1+k\left(y_{1}\right)\left(y-y_{1}\right) / G^{\prime}(y)\right] .
\end{aligned}
$$

Now $y>y_{2}$, so that $G(y) \geqslant G\left(y_{2}\right)$, and for $s \leqslant y_{1}, G(s) \leqslant G\left(y_{1}\right)$. Thus

$$
G(y)-G(s) \geqslant G\left(y_{2}\right)-G\left(y_{1}\right)>0 \text {. }
$$

Consequently

$$
\int_{0}^{y_{1}}[G(y)-G(s)]^{-1 / 2} d s \leqslant y_{1}\left[G\left(y_{2}\right)-G\left(y_{1}\right)\right]^{-1 / 2}=l\left(y_{1}, y_{2}\right) .
$$

From this the second inequality follows.

To prove the last part of the lemma we suppose that $\varepsilon>0$; then we choose $y_{1}>1 / \alpha-1$ such that $\left(\forall y \in\left[y_{1}, 1 / \alpha\right]\right)$;

$$
\left|\left(\frac{1}{2} \alpha e^{1 / \alpha}\right)^{1 / 2} /\left(\frac{1}{2} k(y)\right)^{1 / 2}-1\right|<\varepsilon .
$$

(This follows since $k$ is continuous and $k(1 / \alpha)=\alpha e^{1 / \alpha}$.)

Now fix $y_{2}$ such that $y_{1}<y_{2}<1 / \alpha$; then we pick $y_{3} \geqslant y_{2}$ so that, for $y \in$ $\left[y_{3}, 1 / \alpha\right)$,

$$
l\left(y_{1}, y_{2}\right)\left(\frac{1}{2} \alpha e^{1 / \alpha}\right)^{1 / 2} / \ln [1 /(1-\alpha y)]<\varepsilon .
$$

Writing $M_{1}=y(\alpha+\alpha y-1)$ and $M_{2}=\left(y-y_{1}\right)\left(\alpha+\alpha y_{1}-1\right) e^{y_{1}-y}$, we now consider the quantities, for $j=1,2$,

$$
\begin{aligned}
\mid \cosh ^{-1}[1 & \left.+M_{J} /(1-\alpha y)\right] / \ln [1 /(1-\alpha y)]-1 \mid \\
& =\left|\ln \left[1-\alpha y+M_{J}+\left(M_{J}^{2}+2 M_{J}(1-\alpha y)\right)^{1 / 2}\right] / \ln [1 /(1-\alpha y)]\right| .
\end{aligned}
$$

Then there exists $y_{4} \geqslant y_{3}$ such that, for $y \in\left[y_{4}, 1 / \alpha\right)$,

$$
\ln [1 /(1-\alpha y)] \geqslant \varepsilon^{-1} \max _{j=1,2}\left\{\left|\ln \left[1-\alpha y+M_{j}+\left(M_{j}^{2}+2 M_{j}(1-\alpha y)\right)^{1 / 2}\right]\right|\right\} \text {. }
$$

So, for $y \geqslant y_{4}$

$$
\begin{array}{r}
\left|\cosh ^{-1}[1+y(\alpha+\alpha y-1) /(1-\alpha y)] / \ln [1 /(1-\alpha y)]-1\right|<\varepsilon, \\
\left|\cosh ^{-1}\left[1+\left(y-y_{1}\right) k\left(y_{1}\right) / G^{\prime}(y)\right] / \ln [1 /(1-\alpha y)]-1\right|<\varepsilon .
\end{array}
$$

Taking $\varepsilon<1$ and substituting all these estimates into our inequalities for $I(y)$ we see that, for $y \in\left[y_{4}, 1 / \alpha\right)$,

$$
\begin{aligned}
1-2 \varepsilon & <(1-\varepsilon)^{2}<\left(\frac{1}{2} \alpha e^{1 / \alpha}\right) I(y) / \ln [1 /(1-\alpha y)] \\
& <(1+\varepsilon)^{2}+\varepsilon<1+4 \varepsilon .
\end{aligned}
$$


Therefore we have

$$
I(y) \sim\left(2 e^{-1 / \alpha} / \alpha\right) \ln [1 /(1-\alpha y)] \text { as } y \rightarrow 1 / \alpha^{-} . \quad \text { Q.E.D. }
$$

In order to obtain more information about the behaviour of $I(y)$ we shall now consider $I^{\prime}(y)$.

LEMMA 2. $I^{\prime}(y)$ exists and equals

$$
\int_{0}^{1} \frac{2 G(y)-2 G(y(1-s))-y\left[G^{\prime}(y)-(1-s) G^{\prime}(y(1-s))\right]}{2[G(y)-G(y(1-s))]^{3 / 2}} d s,
$$

for $y \in(0,1 / \alpha)$.

Proof. We first note that

$$
I(y)=y \int_{0}^{1}[G(y)-G(y(1-s))]^{-1 / 2} d s .
$$

Then by considering the difference quotient $(I(y+h)-I(y)) / h$ and using the Lebesgue dominated convergence theorem, we obtain the result.

For the rest of this section we shall write

$$
\gamma(y, s)=2 G(y)-2 G(y(1-s))-y\left[G^{\prime}(y)-(1-s) G^{\prime}(y(1-s))\right] .
$$

So, for $y \in(0,1 / \alpha)$,

$$
I^{\prime}(y)=\frac{1}{2} \int_{0}^{1} \gamma(y, s)[G(y)-G(y(1-s))]^{-3 / 2} d s .
$$

We now proceed to find some results on the sign of $I^{\prime}(y)$.

LEMMA 3. If $\alpha>\frac{1}{4}$ then

$$
(\forall y \in(0,1 / \alpha)) \quad I^{\prime}(y)>0 .
$$

Proof. Firstly we have

$$
\frac{\partial}{\partial s} \gamma(y, s)=y e^{y(1-s)}\left[1-(1-s) y+\alpha(1-s)^{2} y^{2}\right],
$$

and $\gamma(y, 0)=0$. Now, for $\alpha \geqslant \frac{1}{4}$,

$$
1-(1-s) y+\alpha(1-s)^{2} y^{2} \geqslant 0
$$

for any values of $s$ and $y$. Therefore, for $\alpha>\frac{1}{4}, y \in(0,1 / \alpha),(\forall s \in[0,1])$ $(\partial / \partial s) \gamma(y, s)>0$, and so $(\forall s \in(0,1]) \gamma(y, s)>0$. Consequently $(\forall y \in(0,1 / \alpha)$ $I^{\prime}(y)>0$. Q.E.D. 
We note that for $\alpha \leqslant \frac{1}{4}$, (24) becomes

$$
\begin{aligned}
\frac{\partial}{\partial s} \gamma(y, s)= & \alpha y e^{y(1-s)}\left[y(1-s)-\frac{1}{2 \alpha}\left(1-(1-4 \alpha)^{1 / 2}\right)\right] \\
& \times\left[y(1-s)-\frac{1}{2 \alpha}\left(1+(1-4 \alpha)^{1 / 2}\right)\right] .
\end{aligned}
$$

LEMMA 4. Suppose $\alpha<\frac{1}{4}$ and $y_{0}$ is the solution of

$$
y G^{\prime}(y)=\frac{1}{2 \alpha}\left(1-(1-4 \alpha)^{1 / 2}\right) G^{\prime}\left(\frac{1}{2 \alpha}\left[1-(1-4 \alpha)^{1 / 2}\right]\right)
$$

which lies in $\left(\left[1-(1-4 \alpha)^{1 / 2}\right] / 2 \alpha, 1 / \alpha\right)$. If

$$
0 \leqslant y \leqslant\left[1-(1-4 \alpha)^{1 / 2}\right] / 2 \alpha \text { or } y_{0} \leqslant y<1 / \alpha,
$$

then

$$
I^{\prime}(y)>0 \text {. }
$$

Proof. We see from (25) that $\partial \gamma / \partial s>0$ if $y \leqslant\left[1-(1-4 \alpha)^{1 / 2}\right] / 2 \alpha$ and $0<s \leqslant 1$. Therefore, since $\gamma(y, 0)=0$,

$$
(\forall s \in(0,1)) \quad \gamma(y, s)>0, \text { and } I^{\prime}(y)>0 .
$$

It also follows from (25) that, for $y>\left[1+(1-4 \alpha)^{1 / 2}\right] / 2 \alpha$,

$$
\begin{gathered}
\partial \gamma / \partial s>0 \text { for } 0<s<1-\left[1+(1-4 \alpha)^{1 / 2}\right] / 2 \alpha y \\
\text { and } 1-\left[1-(1-4 \alpha)^{1 / 2}\right] / 2 \alpha y<s<1,
\end{gathered}
$$

$\partial \gamma / \partial s<0$ for $1-\left[1+(1-4 \alpha)^{1 / 2}\right] / 2 \alpha y<s<1-\left[1-(1-4 \alpha)^{1 / 2}\right] / 2 \alpha y$.

Consequently $\gamma$ achieves its minimum on $[0,1]$ at either $s=0$ or $s=1-[1-(1$ $\left.-4 \alpha)^{1 / 2}\right] / 2 \alpha y$.

Now,

$$
\begin{aligned}
\gamma\left(y, 1-\left[1-(1-4 \alpha)^{1 / 2}\right]\right. & / 2 \alpha y) \\
= & 2 G(y)-2 G\left(\left[1-(1-4 \alpha)^{1 / 2}\right] / 2 \alpha\right)-y G^{\prime}(y) \\
& +\frac{1}{2 \alpha}\left[1-(1-4 \alpha)^{1 / 2}\right] G^{\prime}\left(\left[1-(1-4 \alpha)^{1 / 2}\right] / 2 \alpha\right)
\end{aligned}
$$

and, since $y>\left[1+(1-4 \alpha)^{1 / 2}\right]$,

$$
G(y)>G\left(\left[1-(1-4 \alpha)^{1 / 2}\right] / 2 \alpha\right) \text {. }
$$

Also, differentiation of $s G^{\prime}(s)$ reveals that $\left[1+(1-4 \alpha)^{1 / 2}\right] / 2 \alpha<y_{0}$ and $s G^{\prime}(s)$ is decreasing for $y_{0} \leqslant s \leqslant 1 / \alpha$. Hence, for $y \geqslant y_{0}$,

$$
\frac{1}{2 \alpha}\left[1-(1-4 \alpha)^{1 / 2}\right] G^{\prime}\left(\frac{1}{2 \alpha}\left[1-(1-4 \alpha)^{1 / 2}\right]\right) \geqslant y G^{\prime}(y)
$$


and $\gamma\left(y, 1-\left[1-(1-4 \alpha)^{1 / 2}\right] / 2 \alpha y\right)>0$. Therefore, for $0<s<1, \gamma(y, s)>0$. Thus, for $y \geqslant y_{0}$,

$$
I^{\prime}(y)>0 \text {. }
$$

LemMA 5. Let $\alpha_{1}=0.224508$; then, for $\alpha \leqslant \alpha_{1}$, there exists some $y_{1} \in(0,1 / \alpha)$ such that, for $y_{1} \leqslant y \leqslant\left[1+(1-4 \alpha)^{1 / 2}\right] / 2 \alpha$,

$$
I^{\prime}(y)<0 \text {. }
$$

PRoof. It follows from (25) that, for $y \in\left(\left[1-(1-4 \alpha)^{1 / 2}\right] / 2 \alpha,[1+(1-\right.$ $\left.\left.4 \alpha)^{1 / 2}\right] / 2 \alpha\right)$,

$$
\begin{array}{ll}
\partial \gamma / \partial s>0 & \text { for } s \in\left(1-\left[1-(1-4 \alpha)^{1 / 2}\right] / 2 \alpha y, 1\right) \\
\partial \gamma / \partial s<0 & \text { for } s \in\left(0,1-\left[1-(1-4 \alpha)^{1 / 2}\right] / 2 \alpha y\right) .
\end{array}
$$

Also,

$$
\begin{aligned}
\gamma\left(\left[1+(1-4 \alpha)^{1 / 2}\right] / 2 \alpha, 1\right) & =2 G\left(\left[1+(1-4 \alpha)^{1 / 2}\right] / 2 \alpha\right)-2 G(0) \\
& -\frac{1}{2 \alpha}\left[1+(1-4 \alpha)^{1 / 2}\right] G^{\prime}\left(\left[1+(1-4 \alpha)^{1 / 2}\right] / 2 \alpha\right) .
\end{aligned}
$$

Now, numerical calculation shows that, for $\alpha \leqslant 0.224508$,

$$
\gamma\left(\left[1+(1-4 \alpha)^{1 / 2}\right] / 2 \alpha, 1\right)<0 .
$$

Since $\gamma$ is continuous there is some $y_{1}$ such that

$$
\left[1-(1-4 \alpha)^{1 / 2}\right] / 2 \alpha<y_{1}<\left[1+(1-4 \alpha)^{1 / 2}\right] / 2 \alpha,
$$

and, for $y \in\left[y_{1},\left[1+(1-4 \alpha)^{1 / 2}\right] / 2 \alpha\right]$,

$$
\gamma\left(y_{1}, 1\right)<0 \text {. }
$$

Using the derivative inequalities (26), we have, for $y_{1} \leqslant y \leqslant[1+(1-$ $\left.4 \alpha)^{1 / 2}\right] / 2 \alpha$ and $0<s \leqslant 1$,

$$
\gamma(y, s)<0
$$

Therefore,

$$
I^{\prime}(y)<0 .
$$

We conclude our discussion of the properties of $I^{\prime}$ by showing its continuity and demonstrating its singular behaviour at the origin.

LEMMA 6. $I^{\prime}(y)$ is continuous on $(0,1 / \alpha)$. 
Proof. Suppose $\left(x_{n}\right)$ is a sequence in $(0,1 / \alpha)$ such that $x_{n} \rightarrow y \in(0,1 / \alpha)$. Then, using the Lebesgue dominated convergence theorem, we can show that $I^{\prime}\left(x_{n}\right) \rightarrow I^{\prime}(y)$ as $n \rightarrow \infty$. Hence $I^{\prime}$ is continuous on $(0,1 / \alpha)$. Q.E.D.

Corollary 7. Let $\alpha_{1}, y_{1}$ be as in Lemma 5, and $y_{0}$ as in Lemma 4. If $\alpha \leqslant \alpha_{1}$ then there exist $X, Y$ such that $I^{\prime}(X)=I^{\prime}(Y)=0$ with

$$
\begin{aligned}
& {\left[1-(1-4 \alpha)^{1 / 2}\right] / 2 \alpha<X<y_{1} \text { and }} \\
& {\left[1+(1-4 \alpha)^{1 / 2}\right] / 2 \alpha<Y<y_{0} .}
\end{aligned}
$$

Proof. This follows from the continuity of $I^{\prime}$ and Lemmas 4 and 5. Q.E.D.

LeMma 8. For any $\alpha>0, I^{\prime}(y) \sim y^{-1 / 2}$, as $y \rightarrow 0^{+}$.

Proof. Firstly,

$$
\begin{aligned}
\gamma(y, s) & =2 G(y)-2 G(y(1-s))-y\left[G^{\prime}(y)-(1-s) G^{\prime}(y(1-s))\right] \\
& =\left[G^{\prime}(y \xi(s))-y \xi(s) G^{\prime \prime}(y \xi(s))\right] y s,
\end{aligned}
$$

where $\xi(s) \in[1-s, 1]$.

Also

$$
G(y)-G(y(1-s))=G^{\prime}(y \zeta(s)) y s,
$$

where $\zeta(s) \in[1-s, 1]$.

So, from Figures 1 and 2, we see that for $y$ near $0(y<1 / \alpha-1$ if $\alpha<1$, or $y \in(0,1 / \alpha)$ if $\alpha \geqslant 1)$ and $s \in[0,1]$,

$$
\begin{aligned}
\gamma(y, s) & \leqslant G^{\prime}(y) y s \text { for } \alpha<1, \\
& \leqslant\left[G^{\prime}(0)-y G^{\prime \prime}(y)\right] \text { ys for } \alpha \geqslant 1,
\end{aligned}
$$

and

$$
\begin{aligned}
G(y)-G(y(1-s)) & \geqslant G^{\prime}(0) y s \text { for } \alpha<1, \\
& \geqslant G^{\prime}(y) y s \text { for } \alpha \geqslant 1 .
\end{aligned}
$$

Therefore, for $\alpha<1$,

$$
I^{\prime}(y) \leqslant \frac{G^{\prime}(y)}{G^{\prime}(0)^{3 / 2} y^{1 / 2}} \int_{0}^{1} \frac{d s}{2 s^{1 / 2}}=\frac{G^{\prime}(y)}{G^{\prime}(0)^{3 / 2}} y^{-1 / 2},
$$


and, for $\alpha \geqslant 1$,

$$
I^{\prime}(y) \leqslant \frac{G^{\prime}(0)-y G^{\prime \prime}(y)}{G^{\prime}(y)^{3 / 2} y^{1 / 2}} \int_{0}^{1} \frac{d s}{2 s^{1 / 2}}=\frac{G^{\prime}(0)-y G^{\prime \prime}(y)}{G^{\prime}(y)^{3 / 2}} y^{-1 / 2} .
$$

Also,

$$
\begin{aligned}
\gamma(y, s) & \geqslant\left[G^{\prime}(0)-y G^{\prime \prime}(y)\right] y s \text { for } \alpha<1, \\
& \geqslant G^{\prime}(y) y s \text { for } \alpha \geqslant 1,
\end{aligned}
$$

and

$$
\begin{aligned}
G(y)-G(y(1-s)) & \leqslant G^{\prime}(y) y s \text { for } \alpha<1, \\
& \leqslant G^{\prime}(0) y s \text { for } \alpha \geqslant 1 .
\end{aligned}
$$

Consequently,

$$
\begin{aligned}
I^{\prime}(y) & \geqslant \frac{G^{\prime}(0)-y G^{\prime \prime}(y)}{G^{\prime}(y)^{3 / 2}} y^{-1 / 2} \text { for } \alpha<1, \\
& \geqslant \frac{G^{\prime}(y) y^{-1 / 2}}{G^{\prime}(0)^{3 / 2}} \quad \text { for } \alpha \geqslant 1 .
\end{aligned}
$$

The result now follows from (27), (28) and (29). Q.E.D.

We can now consider the implications of these results for the bifurcation diagram of (20), (22) (that is a graph of a "norm of the solutions" of (20), (22) against $\lambda$ ). We have shown that

$$
\lambda=\frac{1}{2}\left[I\left(u_{0}\right)\right]^{2},
$$

where $u_{0}=u(0)$. Now, if $u_{1}$ and $u_{2}$ are two solutions of (20), (22) with $u_{1}(0)=$ $u_{2}(0)=u_{0}$ then $u_{1}$ and $u_{2}$ are solutions of the initial value problem

$$
\begin{aligned}
u^{\prime \prime}+\lambda(1-\alpha u) e^{u}=0 & \text { in }(0,1), \\
u^{\prime}(0)=0 & \text { and } u(0)=u_{0} .
\end{aligned}
$$

Consequently $u_{1}=u_{2}$; so $u_{0}=u(0)$ is a suitable norm for the bifurcation diagram of (20), (22).

In Lemma 8 we showed that $I^{\prime}(y) \sim y^{-1 / 2}$ as $y \rightarrow 0^{+}$, so $I(y) \sim 2 y^{1 / 2}$ as $y \rightarrow 0^{+}$. Therefore $\lambda \sim 2 u_{0}$ as $u_{0} \rightarrow 0^{+}$. Now, the other properties of $I$ which we have shown depend on the value of $\alpha$. So we will consider two cases:

(i) $\alpha>\frac{1}{4}$. In this case, by Lemma $3, I^{\prime}(y)>0$ for any $y \in(0,1 / \alpha)$, and so, by Lemma 1 ,

$$
u_{0} \rightarrow 1 / \alpha \text { as } \lambda \rightarrow \infty .
$$

Thus the bifurcation diagram is as in Figure 3. 


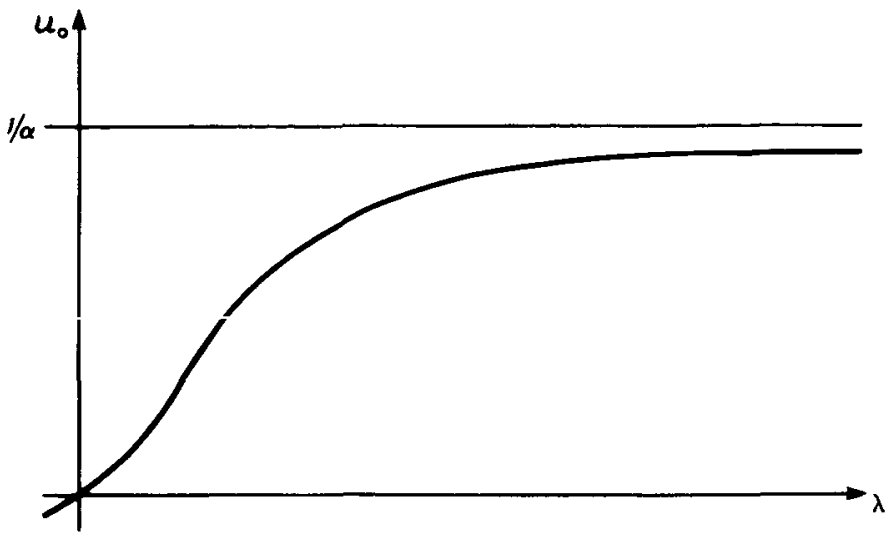

FIGURE 3. Bifurcation diagram for the slab, $\alpha \geqslant \frac{1}{4}$.

(ii) For $\alpha \leqslant \alpha_{1}=0.224508, I^{\prime}(y)$ is negative for some values of $y$. So there is a range of $u_{0}$ for which $\lambda$ decreases with $u_{0}$. Also, $I^{\prime}(y)>0$ for $y$ close to $1 / \alpha$ (Lemma 4); thus, by Lemma $1, u_{0} \rightarrow 1 / \alpha$ as $\lambda \rightarrow \infty$. This leads to a bifurcation diagram similar to that of Figure 4 . We cannot show at this stage that there are only two turning points, however we believe that this is the case. This is represented on the diagram by the dotted part of the curve.

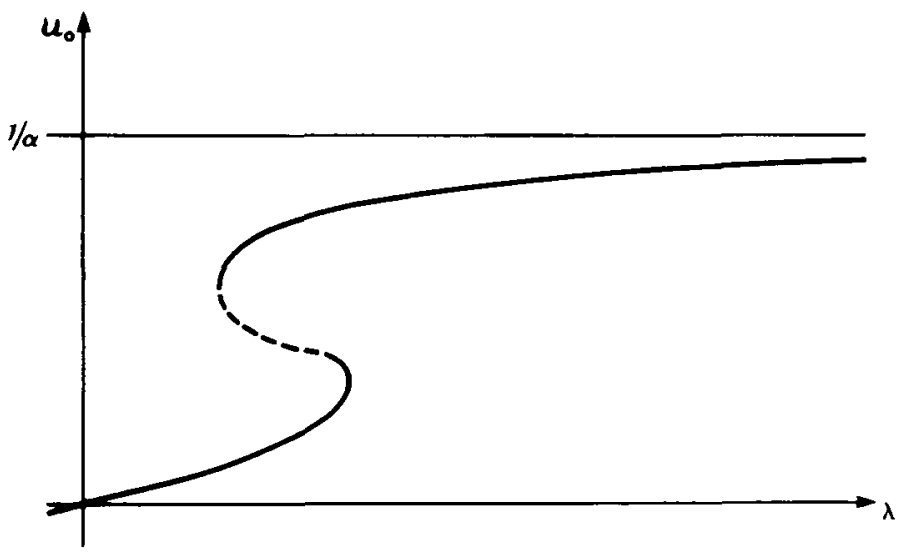

FIGURE 4. Bifurcation diagram for the slab, $\alpha \leqslant \alpha_{1}$.

We see from these diagrams that there must be some transitional value of $\alpha, \alpha_{\mathrm{tr}}$ say, which is the least upper bound of the values of $\alpha$ for which (20), (22) can have multiple solutions for some values of $\lambda$. Then, for $\alpha>\alpha_{\mathrm{tr}}$, the bifurcation diagram always has positive slope, but there are values of $\alpha$, less than and arbitrarily close to $\alpha_{\mathrm{tr}}$, such that the diagram has negative slope somewhere. 
Numerical calculation of $I^{\prime}$ shows that $\alpha_{\mathrm{tr}} \cong 0.242$. Also, we found that, if $\alpha>0.242$ then $I^{\prime}(y)>0$ for all $y \in(0,1 / \alpha)$, while if $\alpha<0.242$ then there is some interval of $y$, depending on $\alpha$, on which $I^{\prime}(y)<0$.

We now turn our attention to a somewhat more general problem, namely the case when $\mu<\nu=\infty$. Here the system (20), (22) can be written in the form (letting $h=\alpha u+v$ )

$$
\begin{gathered}
v^{\prime \prime}-\lambda \alpha(1+v) \exp ((h-v) / \alpha)=0 \quad \text { in }(0,1), \\
v^{\prime}(0)=v(1)=0, \\
h^{\prime \prime}=0 \quad \text { in }(0,1), \\
h^{\prime}(0)=0, \quad h^{\prime}(1)+\mu h(1)=v^{\prime}(1) .
\end{gathered}
$$

By (30b), $h$ is constant on $[0,1], h=B$ say; then

$$
v^{\prime}(1)=\mu B \text {. }
$$

The first integral of (30a) gives

$$
v^{\prime}=\left(2 \lambda \alpha^{2} e^{B / \alpha}\left[G\left(-v_{0} / \alpha\right)-G(-v / \alpha)\right]\right)^{1 / 2}
$$

where $v_{0}=v(0)$ is the minimum value of $v$ on $[0,1]$. So, when $x=1$,

$$
\left(2 \lambda e^{B / \alpha}\right)^{1 / 2}=\mu B / \alpha\left[G\left(-v_{0} / \alpha\right)-G(0)\right]^{1 / 2} \text {. }
$$

Also, the second integral of (30a) yields

$$
\begin{aligned}
\left(2 \lambda e^{B / \alpha}\right)^{1 / 2} & =\int_{v_{0}}^{0} \frac{1}{\alpha}\left[G\left(-v_{0} / \alpha\right)-G(-v / \alpha)\right]^{-1 / 2} d v \\
& =\int_{0}^{-v_{0} / \alpha}\left[G\left(-v_{0} / \alpha\right)-G(t)\right]^{-1 / 2} d t \\
& =I\left(-v_{0} / \alpha\right) .
\end{aligned}
$$

Hence, from (31),

$$
B / \alpha=I\left(-v_{0} / \alpha\right)\left[G\left(-v_{0} / \alpha\right)-G(0)\right]^{1 / 2} / \mu,
$$

and finally, substituting back into (32),

$$
\begin{aligned}
\lambda & =\frac{1}{2}\left[I\left(-v_{0} / \alpha\right)\right]^{2} \exp \left[-I\left(-v_{0} / \alpha\right)\left(G\left(-v_{0} / \alpha\right)-G(0)\right)^{1 / 2} / \mu\right] \\
& =\frac{1}{2} \Gamma\left(-v_{0} / \alpha\right)^{2},
\end{aligned}
$$

where

$$
\Gamma(y)=I(y) \exp \left[-I(y)(G(y)-G(0))^{1 / 2} / 2 \mu\right] .
$$


The properties of $I(y)$, determined in the previous lemmas, can now be used to easily show the following behaviour of $\Gamma(y)$ :

LEMMA 9. $\Gamma(y)$ is a positive function defined and continuous on $[0,1 / \alpha)$ and differentiable on $(0,1 / \alpha)$. Further

$$
\Gamma(0)=0, \lim _{y \rightarrow ! \sim^{-}} \Gamma(y)=0,
$$

and $\Gamma$ achieves its maximum value at some $y^{*} \in(0,1 / \alpha)$.

We shall now show that we can choose $\alpha$ and $\mu$ to ensure that $\Gamma(y)$ has three turning points in $(0,1 / \alpha)$.

Lemma 10. Suppose $\alpha \leqslant 0.224508$; then we define $x_{1}, x_{2}, x_{3}, x_{4}$ and $K(\alpha)$ as follows:

$$
\begin{aligned}
x_{2} & =\min \left\{x \in(0,1 / \alpha): I^{\prime}(x)=0\right\}, \\
x_{3} & =\max \left\{x \in(0,1 / \alpha): I^{\prime}(x)=0\right\}, \\
K(\alpha) & =-\min \left\{I^{\prime}(x): x \in(0,1 / \alpha)\right\}, \\
x_{1} & =\max \left\{x \in\left(0, x_{2}\right): I^{\prime}(x)=K(\alpha)\right\}, \\
x_{4} & =\min \left\{x \in\left(x_{3}, 1 / \alpha\right): I^{\prime}(x)=K(\alpha)\right\} .
\end{aligned}
$$

Also, we define

$$
P(y)=\frac{1}{4} G^{\prime}(y)[I(y)]^{2}[G(y)-G(0)]^{-1 / 2}+\frac{1}{2} I(y) I^{\prime}(y)[G(y)-G(0)]^{1 / 2},
$$

and $L(\alpha)=\sup \left\{|P(y)|: y \in\left(x_{1}, x_{4}\right)\right\}$.

If $\mu_{0}=L(\alpha) / K(\alpha)$ and $\mu>\mu_{0}$ then there exist $y_{1}, y_{2}, y_{3} \in(0,1 / \alpha)$ such that

$$
\Gamma^{\prime}\left(y_{1}\right)=\Gamma^{\prime}\left(y_{2}\right)=\Gamma^{\prime}\left(y_{3}\right)=0 .
$$

Proof. By Lemmas 4, 5, 6 and Corollary $7, x_{2}, x_{3}$, and $K(\alpha)$ are well-defined, and $K(\alpha)$ is seen to be positive. Also, by Lemmas 1 and $8, x_{1}$ and $x_{4}$ are well-defined. Now, by Lemma $6, P(y)$ is seen to be continuous, and hence bounded, on $\left[x_{1}, x_{4}\right]$; thus $L(\alpha)$ is well-defined.

From (35),

$$
\Gamma^{\prime}(y)=\left[I^{\prime}(y)-P(y) / \mu\right] \exp \left\{-I(y)[G(y)-G(0)]^{1 / 2} / 2 \mu\right\} .
$$

Hence, for $\mu>\mu_{0}$,

$$
I^{\prime}\left(x_{1}\right)-P\left(x_{1}\right) / \mu \geqslant K(\alpha)-L(\alpha) / \mu>0,
$$

so $\Gamma^{\prime}\left(x_{1}\right)>0$.

Similarly $\Gamma^{\prime}\left(x_{4}\right)>0$. 
Now, let $x_{5}$ be a point in $\left(x_{2}, x_{3}\right)$ at which $I^{\prime}(y)$ achieves its minimum; so $I^{\prime}\left(x_{5}\right)=-K(\alpha)$. Then

$$
I^{\prime}\left(x_{5}\right)-P\left(x_{5}\right) / \mu \leqslant-K(\alpha)+L(\alpha) / \mu<0 .
$$

Hence $\Gamma^{\prime}\left(x_{5}\right)<0$.

From the continuity of $I^{\prime}(y)$ on $(0,1 / \alpha)$ (Lemma 6) we deduce that $\Gamma^{\prime}(y)$ is also continuous on $(0,1 / \boldsymbol{\alpha})$. Thus, there exist $y_{1} \in\left(x_{1}, x_{5}\right)$ and $y_{2} \in\left(x_{5}, x_{4}\right)$ such that $\Gamma^{\prime}\left(y_{1}\right)=\Gamma^{\prime}\left(y_{2}\right)=0$. Since $\Gamma\left(x_{4}\right)>0$ and $\Gamma(y) \rightarrow 0$ as $y \rightarrow 1 / \alpha^{-}$, it follows that there exists $y_{3} \in\left(x_{4}, 1 / \alpha\right)$ such that $\Gamma^{\prime}\left(y_{3}\right)=0$. Q.E.D.

This lemma enables us to deduce that the bifurcation diagram in this case is similar to that in Figure 5. Here $-v_{0}=-v(0)$ is a suitable norm for the diagram. We note that we have not shown that there are only three turning points, but we believe this to be the case. This is represented on the diagram by the dotted part of the curve.

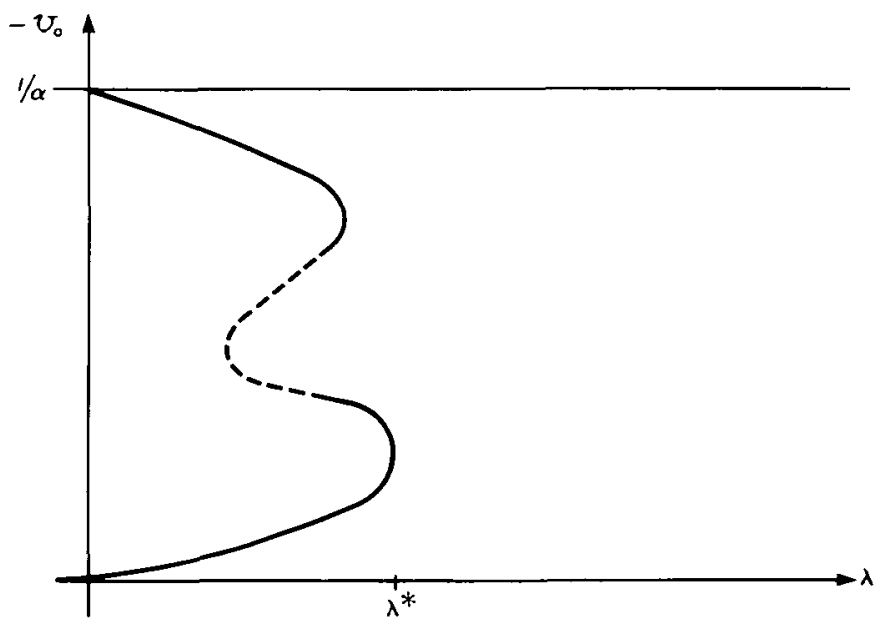

FIGURE 5. Bifurcation diagram for the slab, $\alpha \leqslant \alpha_{1}, \mu>\mu_{0}$.

We note here $\lambda$ has a maximum value $\lambda^{*}$, beyond which there are no solutions to (27), or, equivalently, to (20), (22) with $\nu=\infty$. (See Part I, Section 3.)

Now, $u=(h-v) / \alpha=(B-v) / \alpha$, so using the relationship (33) between $B$ and $v_{0}$, we can use $u_{0}=u(0)$ as a norm in the bifurcation diagram. In this case the diagram is similar to Figure 6. Once again the dotted part of the curve represents our speculations only. 


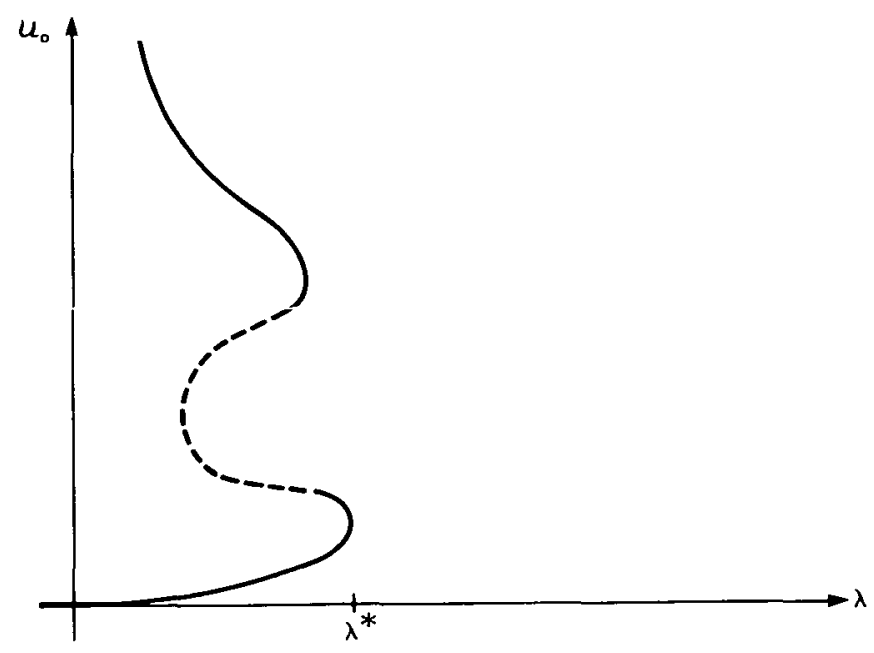

FIgURE 6. Bifurcation diagram for the slab, $\alpha<\alpha_{1}, \mu>\mu_{0}$.

\section{Discussion}

The results in Section 2 on the uniqueness of solutions to the problem (1), (2), together with the results on the existence and continuity of solution branches in Part I (Burnell, Lacey and Wake [3]) give a partial description of the solution set for these steady state equations. Further, these results lead us to conjecture about the behaviour of solutions to the time-dependent problem

$$
\begin{gathered}
\nabla^{2} u+\lambda(1+v) e^{u}=\partial u / \partial t \text { for } x \in \Omega \text { and } t>0 \\
\nabla^{2} v-\lambda \alpha(1+v) e^{u}=\partial v / \partial t \text { for } x \in \Omega \text { and } t>0
\end{gathered}
$$

with boundary conditions in (2) together with prescribed initial conditions.

When there are multiple solutions to (1), (2) then there is a possibility, as shown for the slab, that there is a jump in the norm of the solution as $\lambda$ increases through some value $\lambda_{1}$. This also suggests the possibility of critical behaviour, with a sudden change in the behaviour of the solution to the initial value problem (36), if there are no periodic solutions to (36) for $\lambda$ in some neighbourhood of $\lambda_{1}$. In fact, if the bifurcation diagram is similar to that of Figure 6 then there appears to be the possibility of having critical behaviour and periodic solutions.

In Section 2 we found that there exist intervals of $\lambda$ for which (1), (2) has a unique solution and on which the solution branch is continuous (see Part I, Section 4). This suggests that there is no critical behaviour displayed by the solutions to (36), for these values of $\lambda$. 
For the case of $\mu=\nu=\infty$ in the slab, we have found estimates of $\alpha_{\mathrm{tr}}$ such that for some values of $\alpha<\alpha_{\mathrm{tr}}$ there are multiple solutions, and hence critical behaviour, while for $\alpha \geqslant \alpha_{\mathrm{tr}}$ the solution is always unique and there can be no critical behaviour. From our computed results for this case, we conjecture that for all $\alpha<\alpha_{\mathrm{tr}}$ (1), (2) has multiple solutions, for some values of $\lambda$. We also conjecture that this situation will hold for other regions $\Omega$ and other values of $\mu$ and $\nu$, except for $\mu<\nu=\infty$.

For the slab when $\mu<\nu=\infty$, we see that, for $\lambda<\lambda^{*}$, there are at least two solutions to (30), and if $\alpha$ is sufficiently small and $\mu$ sufficiently large then there is an interval of $\lambda$ in which there are at least four solutions. Taking such values of $\alpha$ and $\mu$, and taking $\nu$ large but finite (so that there must be a solution to (20), (22) for all $\lambda$ ), and since we expect to have solutions close to those for $\nu=\infty$ when $\lambda<\lambda^{*}$, this suggests the possibility of a bifurcation diagram as in Figure 7 .

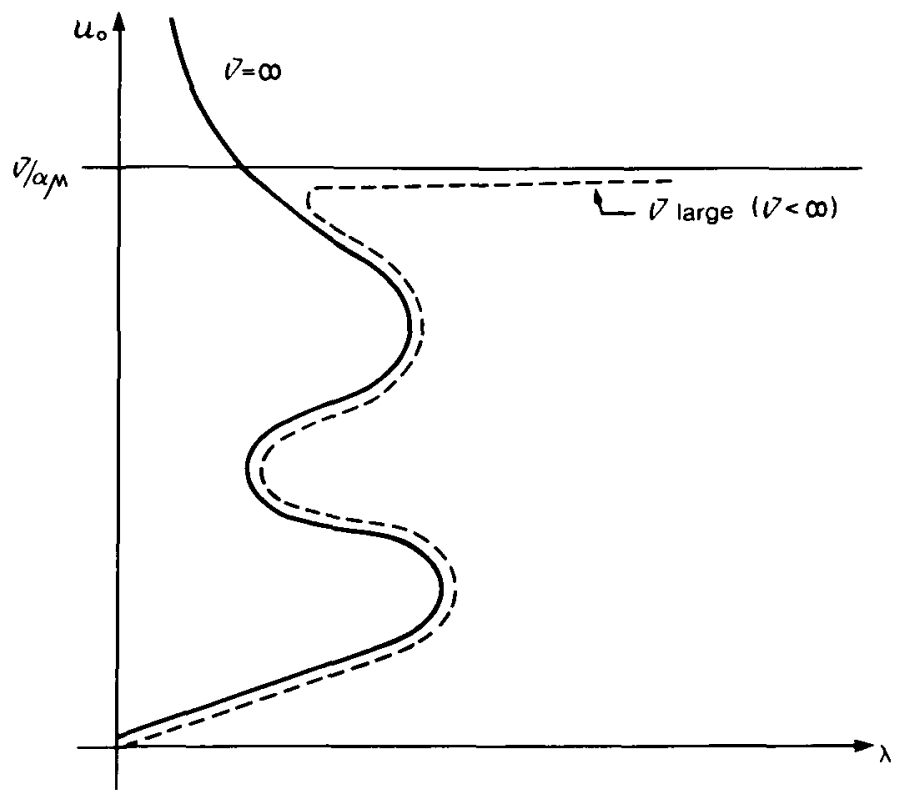

FIGURE 7. Speculative bifurcation diagram for small $\alpha$ and large $\mu$.

Finally, we believe that there will be similar diagrams for more general regions in $\mathbf{R}^{2}$ and $\mathbf{R}^{3}$. 


\section{References}

[1] H. Amann, "Fixed point equations and non-linear eigenvalue problems in ordered Banach spaces", SIAM Rev. 18 (1976), 620-709.

[2] R. Aris, The mathematical theory of diffusion and reaction in permeable catalysts, Vol. 1 (Oxford University Press, 1975).

[3] J. G. Burnell, A. A. Lacey and G. C. Wake, "Steady-states of the reaction-diffusion equations, Part I: Questions of existence and continuity of solution branches", J. Austral. Math. Soc. Ser. B 24 (1983). 375-392.

[4] H. B. Keller and D. S. Cohen, "Some positone problems suggested by nonlinear heat generation", J. Math. Mech. 16 (1967), 1361-1376.

[5] D. H. Sattinger, "Topics in stability and bifurcation theory", Lecture Notes in Mathematics 309 (Springer Verlag, New York, 1973). 